\title{
Influenza surveillance using data from a telemedicine centre
}

\author{
Eva Blozik - Chantal Grandchamp • \\ Jan von Overbeck
}

Received: 6 October 2010/Revised: 14 December 2010/Accepted: 19 January 2011/Published online: 12 February 2011

(C) Swiss School of Public Health 2011

\begin{abstract}
Objectives This study aimed at investigating whether data from medical teleconsultations may contribute to influenza surveillance.

Methods International Classification of Primary Care 2nd Edition (ICPC-2) codes were used to analyse the proportion of teleconsultations due to influenza-related symptoms. Results were compared with the weekly Swiss Sentinel reports.

Results When using the ICPC-2 code for fever we could reproduce the seasonal influenza peaks of the winter seasons $07 / 08,08 / 09$ and $09 / 10$ as depicted by the Sentinel data. For the pandemic influenza 09/10, we detected a much higher first peak in summer 2009 which correlated with a potential underreporting in the Sentinel system.

Conclusions ICPC-2 data from medical teleconsultations allows influenza surveillance in real time and correlates very well with the Swiss Sentinel system.
\end{abstract}

Keywords Influenza surveillance - Seasonal influenza · Pandemic influenza $\cdot$ Telemedicine $\cdot$ Teleconsultation

\section{Introduction}

Seasonal and pandemic influenza waves cause significant morbidity and mortality, especially in higher risk populations (World Health Organisation WHO 2010a). In most countries influenza surveillance systems have been

E. Blozik $(\bowtie) \cdot$ C. Grandchamp · J. von Overbeck

Swiss Centre for Telemedicine Medgate, Gellertstrasse 19, 4020 Basel, Switzerland

e-mail: eva.blozik@medgate.ch developed with two aims: first to enable the early detection of epidemics and second to provide estimates of the morbidity, mortality, and economic impact of influenza waves (Carrat et al. 1998).

In addition to global surveillance activities coordinated by the World Health Organisation (WHO), many countries have national surveillance systems in place. National surveillance provides the most complete information of the extent and severity of influenza activity, the characteristics of the circulating strains, and the clinical effectiveness of the vaccine (Cox et al. 1994; Brammer et al. 2002).

The influenza surveillance system in Switzerland is based on the Swiss Sentinel System (www.sentinella.ch) including about 200 primary care physicians in private practices. The participating practitioners report consultations due to influenza-like illness (ILI) on a weekly basis. Additionally, throat-swaps of a subset of patients are examined at the National Centre of Influenza in Geneva in order to detect and isolate influenza viruses. The assessment of the current activity of influenza is based on both the weekly Sentinel reports of ILI and the detection of influenza viruses by the laboratory (Swiss Federal Office of Public Health SFOPH 2010a).

Internationally, various attempts have been made to use data from other sources such as over-the-counter sales or call centre services for influenza surveillance in addition to sentinel or virus isolation data (Cooper et al. 2002; Das et al. 2005; Yih et al. 2009). However, to our knowledge, no previous study investigated whether clinical data from teleconsultations done by medical doctors could contribute to influenza surveillance. This study aims to evaluate whether the course of influenza waves in the past 3 years as detected by the Swiss Sentinel system can be reproduced by medical teleconsultation data from a large telemedicine centre covering all parts of the country. 


\section{Methods}

\section{Context of the evaluation}

The Swiss Centre for Telemedicine Medgate is the largest telemedicine centre in Europe, currently providing up to 4,300 patient contacts per day. Teleconsultations cover the whole spectrum of medical questions from acute health problems to health behaviour questions. Teleconsultation processes of the centre conform to the American Telemedicine Association's Core Standards for Telemedicine Operations (American Telemedicine Association ATA 2007). Data safety and data protection are guaranteed by specific staff training, quality assurance, and by the technical equipment. Health insurance is compulsory for all persons residing in Switzerland and covers treatment in case of illness or accident (unless another accident insurance provides the cover) and pregnancy. Within the Swiss healthcare system, patients are generally free to choose a face-to-face healthcare provider from their region. This also applied to persons who had access to Medgate teleconsultation services. The Swiss healthcare system is a combination of public (e.g. university hospitals), subsidised private (e.g. home care services), and totally private systems (e.g. doctors in private practice and in private clinics). In contrast to face-to-face consultations, there are no co-payments for a teleconsultation. All insured persons of a health insurance have unrestricted access if the insurance contracted Medgate for teleconsultation services (e.g., for 2010, 3 of the 5 major health insurances; for the total study period about $30 \%$ of all insured in Switzerland). Each resident in Switzerland has access to all registered health insurances so that persons who called for teleconsultations lived in communities spread throughout the country including the four culturally different language regions. The telemedicine centre is paid directly by the health insurers on a per capita basis. The teleconsultation services are available $24 \mathrm{~h}$ a day.

When a patient calls, a telemedical assistant identifies the patient and records the medical problem, makes an assessment of its urgency (based on internal quality standards and under medical supervision), and assigns a time interval during which a physician trained in telemedicine shall call back the patient for a detailed teleconsultation. After a careful medical assessment, the physician recommends either a face-to-face consultation with the appropriate healthcare provider in the appropriate time interval, or selfcare measures. The latter is done in clinical situations in which, according to this assessment, no immediate face-to-face consultation is needed, and includes measures such as bed rest, taking medication, or watchful waiting. The physician instructs the patient regarding alarm symptoms which, should they occur, indicate an immediate need for contacting the telemedicine centre again, or turning to another healthcare provider.

After each teleconsultation, the reasons for encounter (symptoms) and the most appropriate medical diagnoses (diagnosis or suspected diagnosis) are routinely encoded using the International Classification of Primary Care 2nd edition (ICPC-2) (Wonca International Classification Committee 2010). This evaluation focuses on teleconsultations done via the telephone, as internet or video technologies are presently only marginally used in Switzerland.

\section{Evaluation design}

ICPC-2 codes of all patients who received a teleconsultation between week 26 in 2007 and week 25 in 2010 were evaluated, yielding the proportion of patients calling each week for the ICPC-2 codes for symptoms or diagnoses that may be used for coding of the diagnosis or suspected diagnosis of influenza. The following ICPC-2 code combinations were selected by a group of clinicians including specialists in internal medicine, public health medicine, and infectiology because they are most likely to be used for coding of influenza or suspected influenza at our centre. Combinations were used to increase sensitivity of the analysis.

1. Fever as key indicator for influenza: A03 (Fever)

2. Fever or diagnosis code for influenza: A03 or R80 (Influenza)

3. Cough as key indicator for influenza: R05 (Cough)

4. Combination of different codes for viral influenza-like infectious disease: A77 (Viral disease other not specified) or R74 (Upper respiratory infection acute) or R80 (Influenza)

5. Combination of influenza diagnosis code and different symptoms indicative of influenza: R80 (Influenza) or A03 (Fever) or R05 (Cough) or R07 (Sneezing/nasal congestion) or R08 (Nose symptom/complaint other) or R21 (Throat symptom/complaint) or R23 (Voice symptom/complaint).

We compared these results with data from the Swiss Sentinel system supplied by the SFOPH. These data included the proportion of ILI-related face-to-face consultations with primary care physicians for each week in the study period. The three influenza seasons covered by the study period (2007-8, 2008-9, 2009-10) were analysed separately and plotted using Microsoft Excel 2003. Comparison of teleconsultation data with Sentinel data was done based on graphical display. Additionally, for comparability with previous research (e.g. Yih et al. 2009) we calculated pairwise Pearson's correlation coefficients between cases per 1,000 consultations in the telemedicine setting and the Sentinel system, separately for each 
influenza season and for the ICPC-2 code combinations described above. Given the epidemiologic fact of a baseline activity of influenza-like infectious diseases during the year, a baseline level was defined by drawing a horizontal line at the number of cases at week 26 (beginning of the seasonal surveillance interval) to week 25 (end of seasonal surveillance interval).

\section{Results}

Comparison of graphical display

Using the ICPC-2 code for fever (A03) the proportion of cases in the telemedical setting corresponded with the influenza activity detected by the Sentinel data. When accounting for a baseline level of fever cases during all seasons, we were able to reproduce the seasonal influenza peaks of the winter seasons 07/08 and 08/09 (see Fig. 1) for the same time interval and with the same shape as compared with the Sentinel data. For the pandemic influenza 09, the shape of the curve derived from our data fitted in general with that of the SFOPH (see Fig. 1). However, we detected a first peak from July to September 2009 (weeks 27-40) with significantly more cases as compared with the Sentinel data.

When we added the ICPC-2 code for influenza (R80) to the code for fever the peaks of the influenza activity seemed to be slightly accentuated, but the changes were not substantial (figure available upon request).

Based on the analysis of graphical displays, the ICPC-2 code for cough (R05) did not turn out to be useful for detecting influenza activity, as the frequency of cough encounters peaked in all three winter seasons independently from influenza peaks (figure available upon request).

Using a combination of codes for viral infections associated with influenza symptoms (upper respiratory infection acute, influenza or viral disease other not otherwise specified) produced a time profile with a roughly comparable shape. However, in quantitative terms the proportion of cases was lower by about 20 per 1,000 consultations in all seasons, and there was much more noise, leading to an unsharp curve (figure available upon request).

Combining the ICPC- 2 code for influenza with codes for symptoms indicative for influenza (fever, cough, sneezing/ nasal congestion, nose symptom/complaint other, throat symptom/complaint, voice symptom/complaint) increased the total number of cases to a baseline activity level of about 100 cases per 1,000 teleconsultations and a maximum peak activity of about 270 cases per 1,000 teleconsultations. However, there was more imprecision due to noise as compared with using the single code of fever (figure available upon request).
Correlation analysis

Correlation analyses between teleconsultation and Sentinel data revealed generally large correlations (range 0.46-0.93). Using the ICPC-2 code for fever (mean correlation coefficient 0.83 ), the combination of fever and influenza (0.87) and the combination of influenza with symptoms indicative of influenza (0.84) all showed very large correlations between teleconsultation and Sentinel data. The combination of viral influenza-like infectious diseases was less strongly correlated with Sentinel influenza cases (0.72). Using the ICPC-2 code for cough revealed to have the weakest correlation with Sentinel data (0.68) (see Table 1).

\section{Discussion}

Our evaluation shows that ICPC-2 codes from teleconsultations done by trained medical doctors are useful for influenza surveillance. The baseline all-season morbidity of fever or other diagnoses or symptoms investigated need to be taken into account when interpreting the influenza activity. In contrast to previous research using data from call centres with non-medical staff (Cooper et al. 2002; Yih et al. 2009), our data were generated by trained medical doctors during teleconsultations and compared with Sentinel data collected in GP practices. Therefore, agreement between Sentinel data and teleconsultations data in this study may be higher as compared with other work (Yih et al. 2009).

Compared with the frequency of consultations by primary care physicians reporting to the Sentinel systems, the relative number of teleconsultations presumably due to influenza was significantly higher, even when accounting for the baseline all-season morbidity. This may be related to a higher sensitivity for detecting suspected influenza cases in the telemedical setting, as the barrier to access telemedical services for sick persons may be lower than that for seeking a face-to-face consultation (Bashshur 1995).

Several limitations need to be considered when interpreting this research. First, one of our methods was to use a simple graphical approach to compare teleconsultation data with Sentinel data. However, given the context, the graphical and therefore visual analysis is one of the most powerful analyses because it allows evaluating the temporal evolution, the shape and the volume of potential influenza cases. Second, data from a single centre in Switzerland were used. However, about a third of the Swiss population including persons from all four language regions in Switzerland had access to our teleconsultation services because their health insurances contracted our centre. Due to this sampling, persons who have access to 
Fig. 1 International

Classification for Primary Care 2nd Edition (ICPC-2) code for fever compared with Sentinel reporting in the influenza seasons 2007/2008, 2008/2009, and 2009/2010; Basel 2010
ICPC-2 A03 (fever) versus Sentinella reporting: $2007 / 2008$

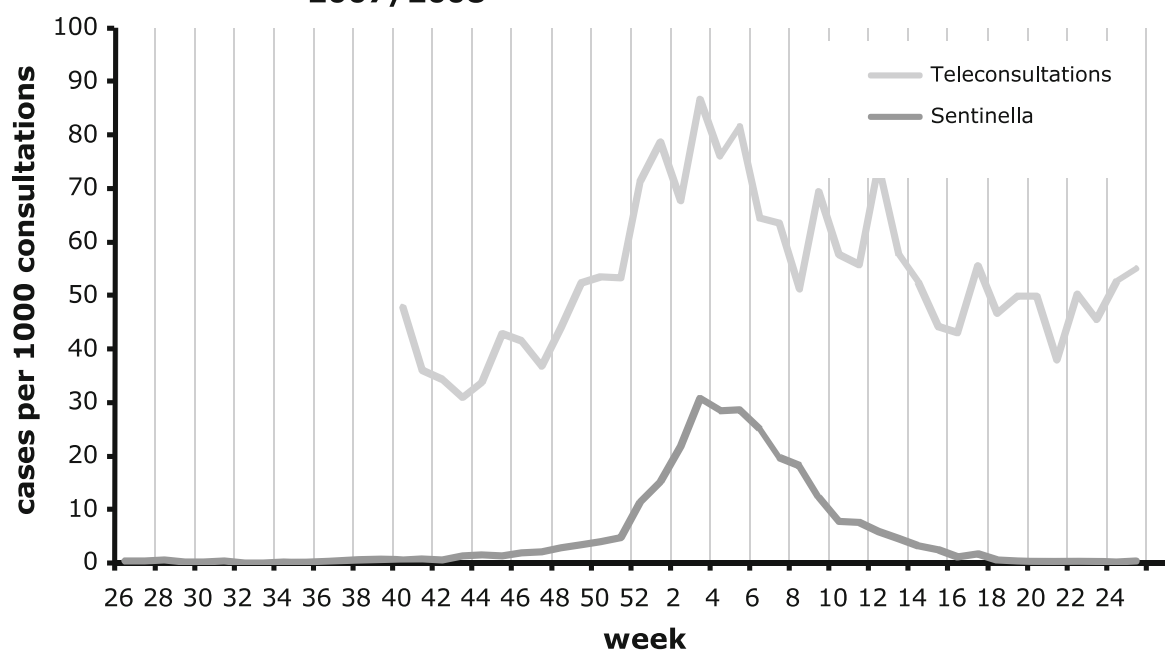

ICPC-2 A03 (fever) versus Sentinella reporting:

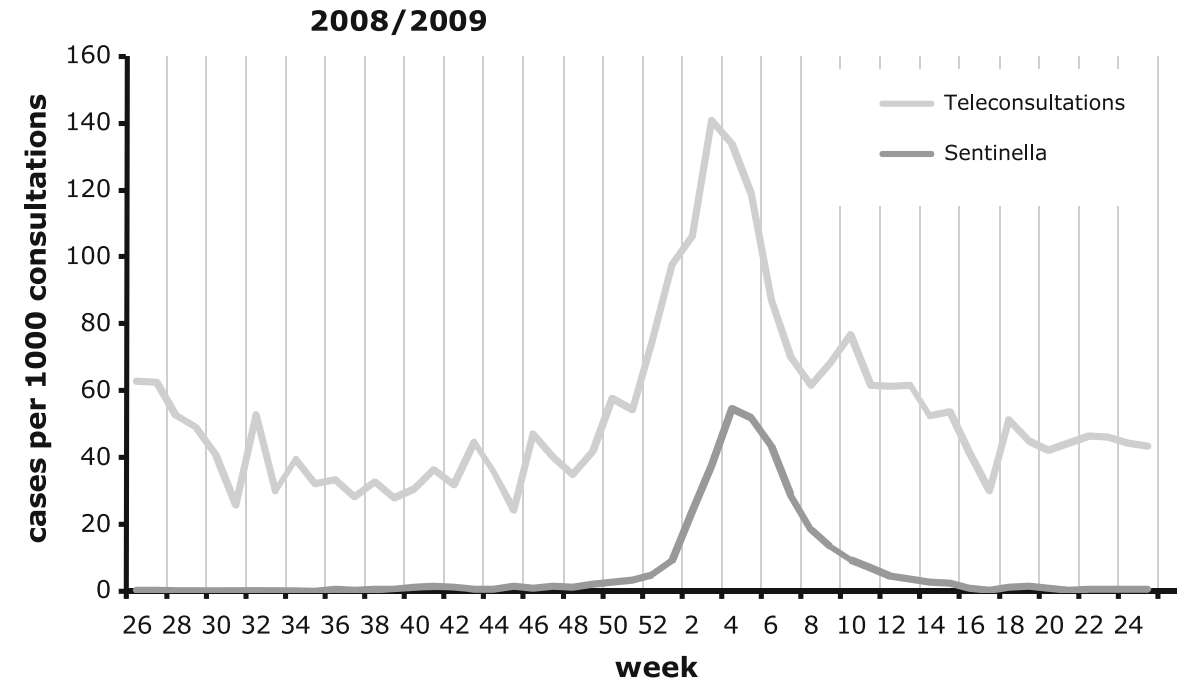

ICPC-2 A03 (fever) versus Sentinella reporting:

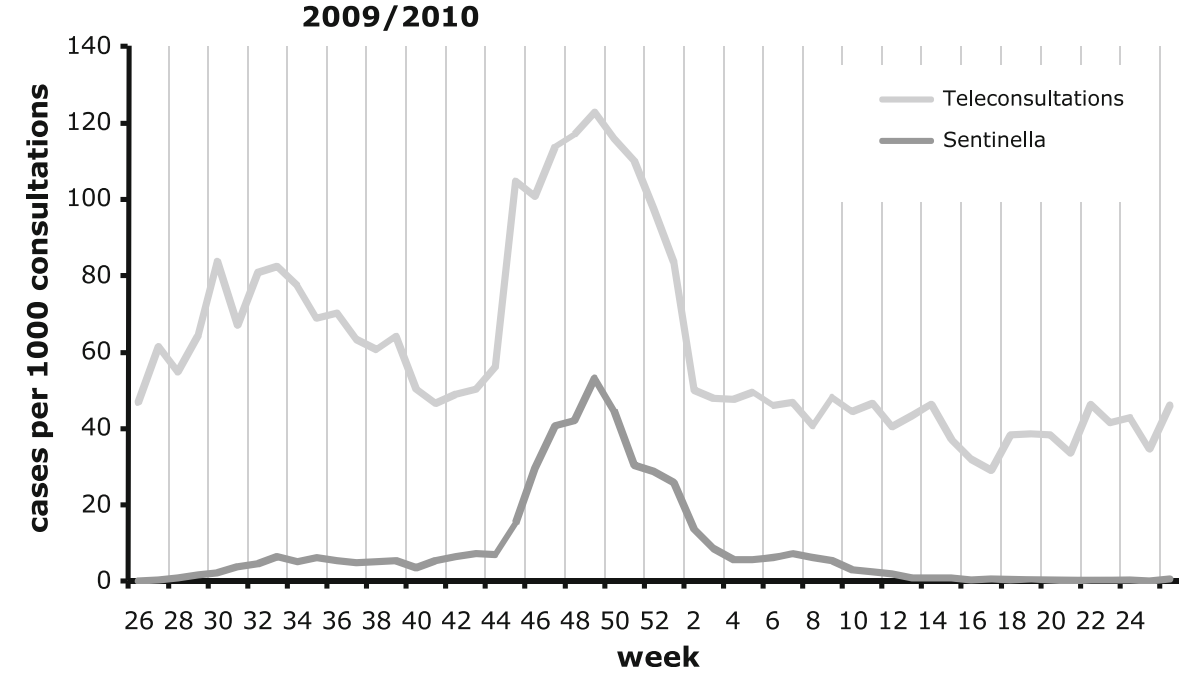


Table 1 Pearson's correlation coefficients between teleconsultation data and Sentinel data

\begin{tabular}{lllll}
\hline ICPC-2 code & $2007 / 2008$ & $2008 / 2009$ & $2009 / 2010$ & Mean \\
\hline A03 & 0.7879 & 0.8472 & 0.8581 & 0.8310 \\
A03 or R80 & 0.7964 & 0.9142 & 0.8948 & 0.8684 \\
R05 & 0.5333 & 0.7105 & 0.7859 & 0.6765 \\
R74 or R80 or A77 & 0.4612 & 0.8541 & 0.8418 & 0.7190 \\
R80 or A03 or R05 or R07 or R08 or R21 or R23 & 0.7280 & 0.8561 & 0.9285 & 0.8375 \\
\hline
\end{tabular}

our services are likely to be representative for the total population of insured in Switzerland. Third, the ICPC data used for analysis was not collected specifically for use in influenza surveillance. Therefore, ICPC coding done by the telemedical personnel at our centre may not be $100 \%$ precise for this objective.

Fourth, persons may call the teleconsultation centre not because of actually presenting a medical symptom but because of being worried and seeking additional information about a symptom. One might argue that the first peak we detected for the pandemic influenza from July to September 2009 in contrast to the Sentinel system was due to a higher level of anxiety and need for information in the population and did not reflect existing influenza symptoms. However, the European Centre for Disease Prevention and Control also detected a first peak during the same time period in their Sentinel virus specimen tests (European Centre for Disease Prevention and Control 2010). At the same time, the system for reimbursement of laboratory services in Switzerland was changed leading to tariff reductions and therefore reduced income of primary care physicians (SFOPH 2010b). This resulted in discussions among primary care physicians as to whether Sentinel influenza reporting to the SFOPH should be stopped, as this agency is responsible for both influenza surveillance and the control of tariffs for laboratory services. These political considerations may well have resulted in incomplete influenza reporting to the SFPOH during this time period.

The strength of our approach is that the data were collected during routine operations without additional financial or time effort representing a simple way to collect data that may be useful for other stakeholders in the Swiss healthcare setting. Additionally, data from teleconsultations are available-in principle-in real-time. There is almost no lag-time as the time needed to compile, transfer, analyse and display teleconsultation data for surveillance purposes is a few hours. In contrast to the weekly Sentinel reports, surveillance reports based on teleconsultation data may be requested on a daily basis.

ICPC codes are recommended by the World Health Organisation (WHO) mainly as a reason for encounter classification in primary care or general practice wherever applicable and is implemented in many countries (World Health Organisation WHO 2010b). Based on the results of this study, using ICPC-2 codes seems to be useful for predicting influenza waves. Based on the graphical display, using the single ICPC-2 code (A03, fever) or a combination of the influenza and fever code gave a time profile which was very similar to the time profile of influenza activity portrayed by the Sentinel data. In correlation analyses, a combination of influenza with symptoms indicative of influenza showed to be equally related to Sentinel cases as compared with the single fever code or the fever/influenza code combination. However, all combinations tested showed large correlations with Sentinel cases underlining the usefulness of such data for influenza surveillance. Future studies should evaluate which code combinations are most useful, and whether alternative coding systems such as the International Classification of Diseases (ICD) are more appropriate for influenza surveillance.

This evaluation has implications for public health practice in Switzerland. In the Swiss healthcare setting, a large number of teleconsultations are done on a routine basis. It would therefore be worthwhile to routinely include teleconsultation data in the national influenza surveillance activities. This would be feasible at low additional cost. Specifically, using teleconsultation data may help to identify early beginnings of an influenza outbreak because surveillance reports would be available with almost no lagtime. Due to the high number of teleconsultations done in Switzerland, the sensitivity to detect influenza outbreaks earlier may be higher because of the larger population observed and because the barrier to access teleconsultation services may be lower as compared to face-to-face consultations.

In conclusion, using data from medical teleconsultations for influenza surveillance is feasible and would support the existing Sentinel reporting systems.

Acknowledgments This study was funded by the Swiss Centre for Telemedicine Medgate, Basel, Switzerland. The Swiss Federal Office for Public Health has kindly provided us with the original data of the Sentinel surveillance system in Switzerland for use in this paper.

Conflict of interest The authors are employed by the Swiss Centre of Telemedicine Medgate in Basel, Switzerland. 


\section{References}

American Telemedicine Association ATA, USA (2007) http://www. americantelemed.org/files/public/standards/CoreStandards_with COVER.pdf. Accessed 8 December 2010

Bashshur RL (1995) Telemedicine effects: cost, quality, and access. J Med Syst 19:81-91

Brammer TL, Murray EL, Fukuda K, Hall HE, Klimov A, Cox NJ (2002) Surveillance for influenza-United States, 1997-98, 1998-99, and 1999-00 seasons. MMWR Surveill Summ 51:1-10

Carrat F, Flahault A, Boussard E, Farran N, Dangoumau L, Valleron AJ (1998) Surveillance of influenza-like illness in France The example of the 1995/1996 epidemic. J Epidemiol Community Health 52(Suppl 1):32S-38S

Cooper DL, Smith GE, Hollyoak VA, Joseph CA, Johnson L, Chaloner R (2002) Use of NHS Direct calls for surveillance of influenza-a second year's experience. Commun Dis Public Health 5:127-131

Cox NJ, Brammer TL, Regnery HL (1994) Influenza: global surveillance for epidemic and pandemic variants. Eur J Epidemiol 10:467-470

Das D, Metzger K, Heffernan R, Balter S, Weiss D, Mostashari F (2005) Monitoring over-the-counter medication sales for early detection of disease outbreaks-New York City. MMWR Morb Mortal Wkly Rep 54:41-46

European Centre for Disease Prevention and Control, Sweden (2010) http://ecdc.europa.eu/en/healthtopics/H1N1/whats_new/Pages/ publications.aspx?page $=1$. Accessed 08 December 2010

Swiss Federal Office of Public Health SFOPH, Switzerland (2010a) http://www.bag.admin.ch/k_m_meldesystem/00736/00816/index. html?lang=en. Accessed 27 September 2010

Swiss Federal Office of Public Health SFOPH, Switzerland (2010b) http://www.bag.admin.ch/themen/krankenversicherung/00263/ 00264/04185/index.html?lang=fr. Accessed 27 September 2010

Wonca International Classification Committee, Trondheim (2010) http://www.globalfamilydoctor.com/wicc/sensi.html. Accessed 03 December 2010

World Health Organisation WHO, Geneva (2010a) http://www. who.int/mediacentre/factsheets/fs211/en/index.html. Accessed 27 September 2010

World Health Organisation WHO, Geneva (2010b) http://www.who. int/classifications/icd/adaptations/icpc2/en/index.html. Accessed 10 December 2010

Yih WK, Teates KS, Abrams A, Kleinman K, Kulldorff M, Pinner R, Harmon R, Wang S, Platt R (2009) Telephone triage service data for detection of influenza-like illness. PLoS One 4:e5260 